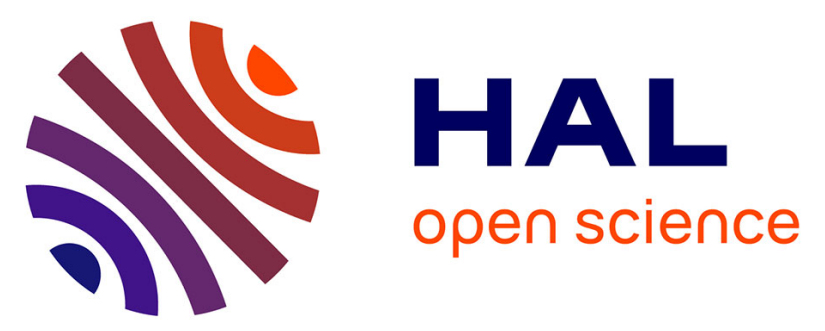

\title{
High temperature rheology of synthetic two-phase gabbroic aggregates: microstructural heterogeneities and local deformation mechanism
}

Jean Raphanel, Alexandre Dimanov, L.A. Nazarova, L.A. Nazarov, A.I. Artemova

\section{To cite this version:}

Jean Raphanel, Alexandre Dimanov, L.A. Nazarova, L.A. Nazarov, A.I. Artemova. High temperature rheology of synthetic two-phase gabbroic aggregates: microstructural heterogeneities and local deformation mechanism. Fiziko-Tekhnicheskie Problemy Razrabotki Poleznykh Iskopaemykh / Journal of Mining Science, 2010, 46 (5), pp.495-502. 10.1007/s10913-010-0062-1 . hal-00641811

\section{HAL Id: hal-00641811 \\ https://hal.science/hal-00641811}

Submitted on 3 Sep 2021

HAL is a multi-disciplinary open access archive for the deposit and dissemination of scientific research documents, whether they are published or not. The documents may come from teaching and research institutions in France or abroad, or from public or private research centers.
L'archive ouverte pluridisciplinaire HAL, est destinée au dépôt et à la diffusion de documents scientifiques de niveau recherche, publiés ou non, émanant des établissements d'enseignement et de recherche français ou étrangers, des laboratoires publics ou privés. 


\title{
HIGH TEMPERATURE RHEOLOGY OF SYNTHETIC TWO-PHASE GABBROIC AGGREGATES: MICROSTRUCTURAL HETEROGENEITIES AND LOCAL DEFORMATION MECHANISM
}

\author{
J. Raphanel, A. Dimanov, L. A. Nazarova*, L. A. Nazarov*, \\ and A. I. Artemova**
}

The high-temperature rheology of heterogeneous anorthite-diopside aggregates has been investigated numerically, in support to experimental data obtained by triaxial torsion tests, performed at high pressure $(400 \mathrm{MPa})$ and temperature $\left(1150^{\circ} \mathrm{C}\right)$. The mechanical data exhibited linear viscous flow. Accordingly, scanning electron microscopy revealed grain sliding mechanisms, but also crystal slip plasticity, recrystallization and micro-fracturing. Finite element computations at the aggregate scale aimed at the understanding of the sequence of active mechanisms and their link to the macroscopic behavior. For instance, we show that the presence of coarser and stronger diopside inclusions in weaker and fine grained anorthite matrix results in very heterogeneous local stress fields, allowing for the activation of multiple deformation mechanisms. Our study indicates that shear zones in the lower crust should be dominated by Newtonian rheology in relation with grain sliding mechanisms, even though complementary accommodation mechanisms such as crystal plasticity and damage may be necessary at the local scale, due to the heterogeneous microstructures.

Rock, laboratory, experiment, high pressure and temperature, equation of state, numerical modeling

\section{INTRODUCTION}

Large-scale geomechanical processes in the upper crust, such as formation and evolution of local stress fields, which govern the choice of a mineral extraction technology, in many ways depend on the globe-scale geodynamics of the lithosphere and astenosphere. To model geodynamical processes related to plate tectonics, it is usual to construct lithosphere strength profiles [1]. The latter depend in depth on the lithosphere structure and mineralogy, on the geothermal gradient and on the corresponding dominant deformation mechanisms. Schematically, the resistance of the cold and fragile upper crust is determined by the frictional sliding behavior of rigid blocks along preexisting faults. Conversely, the resistance of the warm and ductile lower crust represents the flow stress corresponding to the dominant crystal plasticity mechanism. Indeed, at high temperatures $\left(T>500^{\circ} \mathrm{C}\right)$ and natural strain rates $\left(10^{-12}-10^{-15} \mathrm{~s}^{-1}\right)$ the crustal constitutive major silicates (quartz, feldspars, plagioclases, clinopyroxenes and amphiboles) are plastic, but depending on the thermodynamic conditions ( $T, P$ volatile fugacities, loading) and the microstructures (i.e. phase fractions and distributions, grain sizes distributions, textures...) of the considered assemblage different individual or interplaying plastic mechanisms my take place. Their respective contribution may also change with the evolution of microstructures and thus with the $T-\mathrm{P}$ path and the stress and strain history. For instance, crystal slip plasticity (CSP) is a major plastic mechanism, which is characterized by highly non-linear behavior (high stress exponent) and grain size insensitivity. CSP relates to dislocation glide and/or climb, and usually results in

Laboratoire de Mécanique des Solides, CNRS UMR 7649, Ecole Polytechnique, Palaiseau, Paris, France.

*Institute of Mining, Siberian Branch, Russian Academy of Sciences, Novosibirsk, Russia. **Novosibirsk State University, Novosibirsk, Russia. 
pronounced microstructural evolution [2] due to dynamic recrystallization (recovery mechanisms driven by elastic strain energy associated with the development of dislocation density and corresponding crystal structure defects) and the development of textures related to crystal preferred orientation. Conversely, grain boundary sliding (GBS) is characterized by linear viscous behavior and pronounced grain size sensitivity. GBS often relates to diffusional mass transfer and/or interfacial dislocations, and is involved in superplastic behavior (e.g. [3, 4]). In opposition to CSP, GBS do not result in spectacular microstructural evolutions. As concerned with the lower crustal, ductile deformation often takes place in highly localized high temperature shear zones (mylonites), where progressively increasing strain results in spectacular microstructural evolution [5,6], which may correspondingly affect the overall mechanical behavior. The strain gradient developed across the shear process zone reveal:

1. Grain size reduction of the host rock by CSP and dynamic recrystallization [2];

2. Phase mixing by grain switching, solution-precipitation and preferential nucleation [6, 7];

3. Further strain localization within the central ultramylonitic zone, where complete phase mixing ensures stabilized small grain size, which favours GBS and correspondingly low flow stress [3].

However, according to the dislocation microstructures (densities, slip bands, sub-grains boundaries) CSP still operates within ultramylonites and is clearly responsible for the marginal recrystallization of the remaining porphyroclasts [6]. Moreover, according to dislocation densities, sub-grain and recrystallized grain sizes flow stresses could have been substantially high [5]. It is therefore difficult to discriminate which mechanism actually dominates, and thus which rheology should characterizes at best the overall behaviour of the shear zone. In a recent experimental work [8] have clearly characterized the stress strain rate conditions at which either CSP or GBS dominates the rheological behaviour of heterogeneous two-phase gabbroic (plagioclase - clinopyroxene) samples representative of lower crustal rocks [9]. For instance, they have shown the existence of a transition zone where both mechanisms contribute equally and where the overall rheology is characterized by a composite type of flow law. On the other hand, they have also shown that the heterogeneous nature of the two-phase assemblages may locally induce inhomogeneous stress fields, with local stress concentrations at the stronger clinopyroxene inclusions (load transfer), and hence local contribution of CSP in spite of global GBS. However, their results did not allow any quantification of the load transfer effect. Besides, the rather low strain achieved in their experiments (co-axial compression) makes it difficult to directly extrapolate the results to high strain shear zones. This is why, considering the same kind of material, we have performed an additional experimental investigation based on torsion deformation allowing for high shear strain and a finite element numerical analysis with specific emphasis on the identification/quantification of the locally active mechanisms in respect with the global mechanical response.

\section{EXPERIMENTAL WORK}

\section{Sample preparation and deformation}

We prepared synthetic gabbroic material composed of $70 \%$ fine-grained (size $d<5 \mu \mathrm{m}$ ) ductile plagioclase matrix containing $30 \%$ coarser $(d<55 \mu \mathrm{m})$ and stronger clinopyroxene inclusions. The plagioclase matrix originates from synthetic anorthite $\left(\mathrm{CaAl}_{2} \mathrm{Si}_{2} \mathrm{O}_{8}\right)$ glass powder, whereas the clinopyroxene dispersion is obtained by milling gem quality diopside single crystals (with $2 \% \mathrm{Fe}$ as major impurity). The material was hot isostatically pressed at $300 \mathrm{MPa}$ and $1150^{\circ} \mathrm{C}$ in steel container $(1.5 \mathrm{~cm}$ in diameter), using a Paterson type gas apparatus [10]. Traces of water $(0.07 \%)$ comparable to those on natural assemblages were quantified by Fourier transform infra red spectroscopy. 
One sample was kept as reference for the starting material and one sample $(10 \mathrm{~mm}$ in diameter and $7 \mathrm{~mm}$ in height) was deformed in torsion at confining pressure $P=370 \mathrm{MPa}$ and $T=1150^{\circ} \mathrm{C}$ using a Paterson apparatus. We performed a constant torque test (torsion creep) at loading conditions chosen to correspond to the GBS regime, as defined by [8]. Figure $1 a$ presents the experimental loading curve, where the raw mechanical data (twist rate and torque) were recalculated in terms of shear strain rate and shear stress according to [10]. Steady state flow was pursued up to shear strain $\gamma=3.4$, at which the sample suddenly failed with no precursor sign (no creep acceleration). The Figure $1 b$ shows a comparison of the torsion data with the previous results from co-axial compression tests of [8]. For this purpose, we converted the shear stress $\tau$ and the shear strain rate $\dot{\gamma}$ respectively to equivalent axial stress $\sigma=\sqrt{3} \tau$ and axial strain rate $\dot{\varepsilon}=\sqrt{3} \dot{\gamma}$ following [10].

\section{Experimental Mechanical Data}

Several previous studies investigated the steady state flow laws for fine-grained anorthite and diopside homogeneous single phase materials [11-13], and for their two-phase mixtures [8, 14]. These flow laws may be represented by a power-law of the general form $[8,12,14]$.

$$
\dot{\varepsilon}=A d^{m} \sigma^{n} e^{-Q / R T},
$$

where $\dot{\varepsilon}$ is the strain rate, $\sigma$ and $n$ are, respectively, the flow stress and the flow stress exponent, $d$ and $m$ are, respectively, the grain size and the grain size exponent, $Q$ is the activation energy, $R$ and $T$ have the usual meanings, $A$ is a material related constant. The parameters $n, m$ and $Q$ are characteristic of the specifically activated micro-physical plastic mechanisms. For instance, pronounced grain size sensitivity $(m=2-3)$ and linear dependence of strain rate on flow stress $(n=1)$ characterize grain boundary sliding (GBS) accommodated by diffusional mass transfer, whilst grain size insensitivity and elevated stress exponents $(n=3-5)$ are characteristic of CSP associated to dislocation climb recovery and dynamic recrystallization $[8,11,12,14]$.
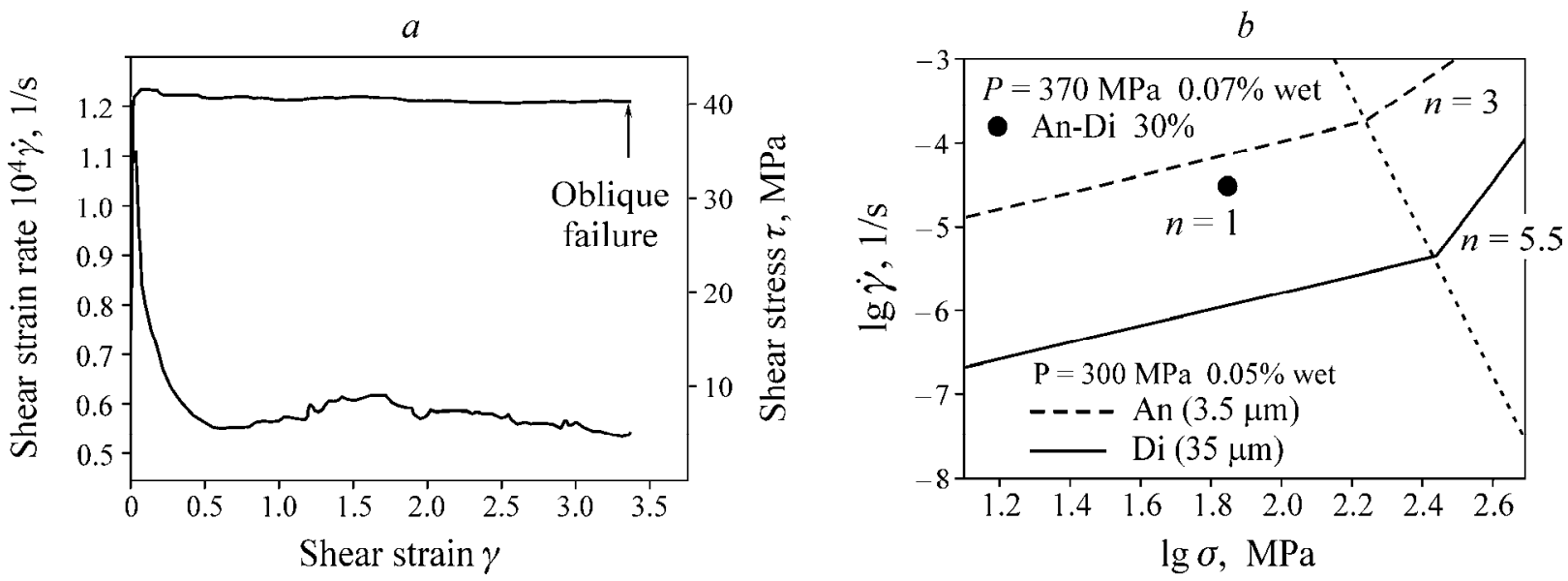

Fig. 1. Mechanical data. (a) Loading curve showing the applied shear stress $\tau$ and the resulting shear strain rate $\dot{\gamma}$ as a function of shear strain $\gamma$. Transient creep is brief: strain rate stabilizes at about $6 \cdot 10^{-5} \mathrm{~s}^{-1}$ for $\gamma>0.5$. Oblique failure suddenly happened at without any precursor sign. (b) Comparison of our data (circle) with previously published flow laws from [8] for end members anorthite (An) and diopside (Di) deformed in tri-axial compression. The flow laws for two-phase mixtures from [8] are bracketed by those for An and Di. GBS regime defined by $n=1$ dominates at low stresses, whilst CSP defined by $n=3$ prevails at higher stresses. Our present data fall within the GBS regime 
Figure $1 b$ shows the comparison of our present result and the flow laws obtained by [8] for pure anorthite and pure diopside (Table below). These previous data correspond to materials comparable to ours, with similar amounts of traces of water, with a mean grain size of $3.5 \mu \mathrm{m}$ for anorthite, but with a smaller grain size $35 \mu \mathrm{m}$ for diopside. The data of [8] evidenced for both phases the presence of two dominant plastic mechanisms acting in parallel and contributing to the constitutive flow law of the form:

$$
\dot{\varepsilon}=A_{\mathrm{GBS}} d^{m} \sigma^{n_{\mathrm{GBS}}} e^{-Q_{\mathrm{GBS}} / R T}+A_{\mathrm{CSP}} \sigma^{n_{\mathrm{CSP}}} e^{-Q_{\mathrm{CSP}} / R T} .
$$

The linear-viscous regime $\left(n_{\mathrm{GBS}}=1\right)$ corresponding to diffusion controlled grain boundary sliding (GBS) was dominant at low stresses $[8,12]$, whilst a highly non-linear behavior $\left(n_{\mathrm{CSP}}=3-5.5\right)$ related to CSP and dislocation creep [8] dominated at higher stresses. The transition between the two mechanisms was not as sharp as it is schematized in Fig. 1, where it is represented by the dot-line. Close to this transition the materials presented a limited transition zone, with mixed type of behavior. Our present data (symbol) for a composite two-phase material consistently falls within the GBS regime for both end members anorthite and diopside.

\section{Microstructures and Identification of Active Micro-Mechanisms}

The cylindrical sample was cut as to produce longitudinal and transverse sections [10] for SEM investigation. Longitudinal sections show the outer most rim of the sample, where can be observed the microstructures corresponding to the highest train in simple shear geometry, whilst transverse sections allow to observe the evolution of microstructures with strain along the sample radius. Figure 2 shows scanning electron microscopy (SEM) micrographs of the outermost rim (maximum strain), which evidence the different operational deformation mechanisms. The grain scale cavitation and coalescence within the fine grained matrix are evidences for dominant grain boundary sliding mechanisms. The slip lines and recrystallization features of the diopside inclusions are signatures of concomitant crystal slip plasticity. In addition, the larger diopside particle are often damaged, but micro-fracturing is strictly limited to the coarser particles and do not extend within the ductile matrix.

The microstructures indicate that the overall flow behavior is ensured by the fine-grained matrix, which is dominated by GBS and exhibits Newtonian like flow. However, dislocation creep is particularly active in the vicinity of and within the stronger diopside particles. Crystal plasticity and damage necessitate substantially higher stresses than the flow stresses supported by the material. This observation indicates that although most the matrix is dominated by GBS and the overall behavior remains Newtonian, the heterogeneous two-phase microstructure generates highly inhomogeneous stress fields allowing for intense local CSP within inclusions and surrounding matrix.

TABLE. Parameters of Ductile Flow Law $\dot{\varepsilon}=A \sigma^{n} e^{-Q / R T}$ for Wet An, Di and An75Di35 from [8]

\begin{tabular}{|c|c|c|c|}
\hline Material & $A, \mathrm{~Pa}^{-n} \mathrm{~s}^{-1}$ & $n$ & $Q, \mathrm{~kJ} / \mathrm{mol}$ \\
\hline $\mathrm{Di}_{35}$ & $\begin{array}{r}1.08 \times 10^{-1^{+}}+6.45 \times 10^{-1} \\
-0.93 \times 10^{-1}\end{array}$ & 1 & $349 \pm 23$ \\
\hline $\mathrm{An}_{75 \mathrm{Di}_{35}}$ & $\begin{array}{r}8.47 \times 10^{-3}+5.58 \times 10^{-2} \\
-7.35 \times 10^{-3} \\
\end{array}$ & 1 & $310 \pm 24$ \\
\hline An & $\begin{array}{r}3.21 \times 10^{-2}+1.37 \times 10^{-1} \\
-2.60 \times 10^{-2} \\
\end{array}$ & 1 & $286 \pm 19$ \\
\hline Di & $\begin{array}{r}5.16 \times 10^{-33^{+}}+.98 \times 10^{-32} \\
-4.85 \times 10^{-33} \\
\end{array}$ & $5.52 \pm 0.09$ & $534 \pm 32$ \\
\hline An75Di & $\begin{array}{r}3.16 \times 10^{-28^{+}+5.56 \times 10^{-27}}-2.99 \times 10^{-28}\end{array}$ & $5.03 \pm 0.07$ & $533 \pm 34$ \\
\hline $\mathrm{An}^{*}$ & $3.981 \times 10_{-1.986 \times 10^{2}}^{2+3.962 \times 10^{2}}$ & $3.00 \pm 0.00$ & $356 \pm 9$ \\
\hline
\end{tabular}

*The dislocation creep flow law for An is from [11], where the $A$ is given in $\mathrm{MPa}^{-n} \mathrm{~s}^{-1}$ 
$a$

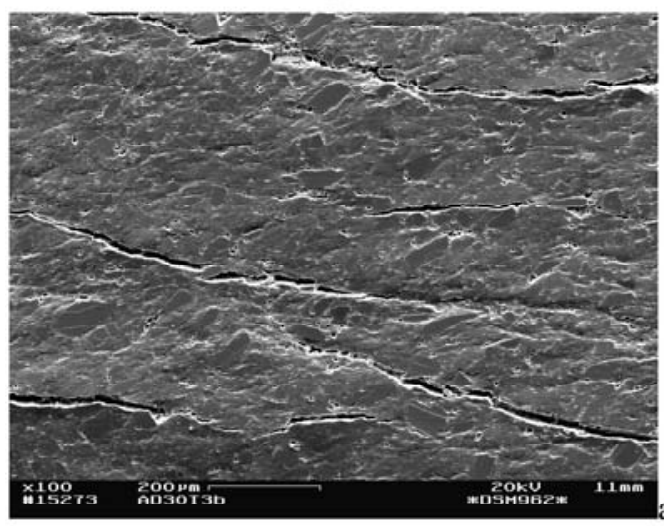

c

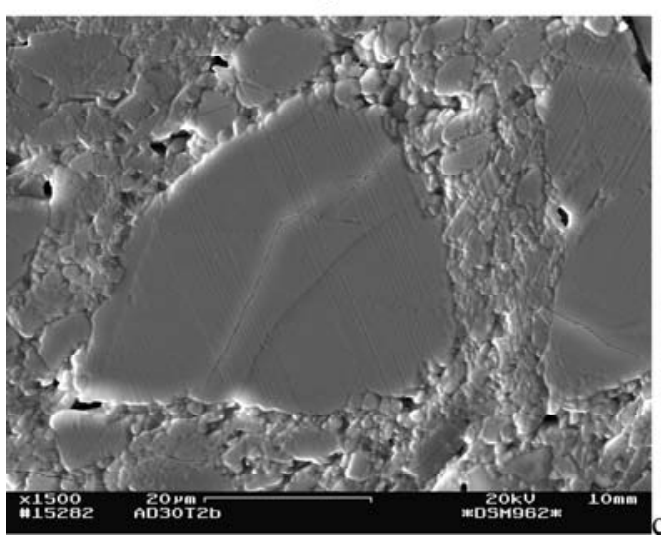

$b$

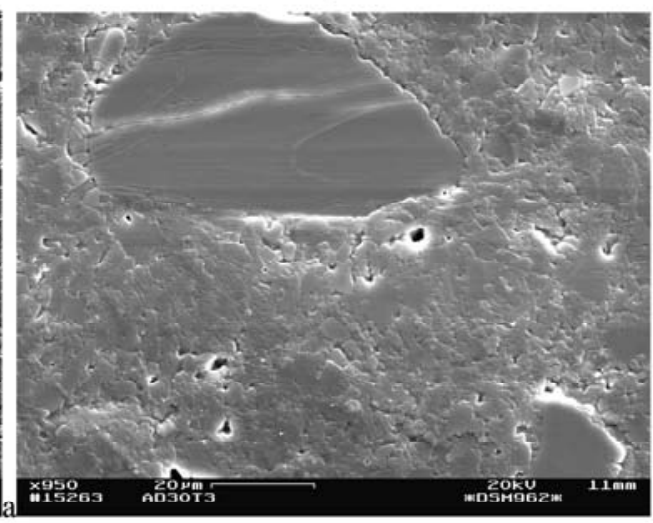

$d$

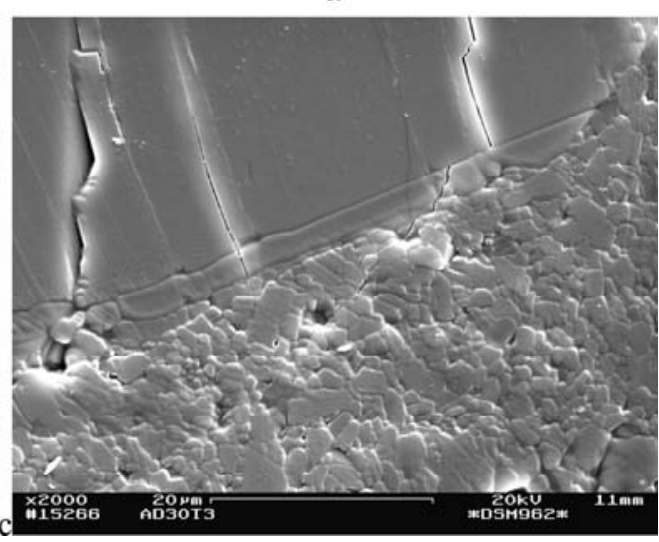

Fig. 2. SEM micrographs from the outer most rim of the twisted specimen (dextral shear: top to the right). Thermal etching reveals grain boundaries of the fine grained matrix and decorates deformation features within the coarse inclusions. (a) Preferential alignment of high aspect ratio inclusions. Cavitation coalescence forming shear bands (c.a. $20^{\circ}$ to the shear plane), leading to micro-cracking and failure. (b) Inclusion damage (cracking is solely limited to the inclusions) and matrix grain scale cavitation. (c) Grain scale cavitation, inclusion damage and crystal plasticity slip lines within inclusions. (d) Inclusion damage and peripheral recrystallization associated with CSP

\section{NUMERICAL SIMULATION}

In order to quantify the local stress heterogeneities indicated by our observations we performed numerical simulations of simple shear with original finite element homebrew codes [15-17]. We considered typical representative elementary volumes (REV, Fig. 3) constituted of anorthite matrix containing irregular diopside inclusions, which assembly is embedded in an effective medium presenting the homogenized bulk properties of a composite with diopside. The inclusions were specifically meshed accounting for the irregular and faceted nature of the starting diopside particles, in order to investigate the effects on the local mechanical fields of inclusion shapes and interactions. We considered elasto-viscoplastic properties for both phases and for the equivalent medium. The Table presents the considered viscoplastic steady state flow laws in both the Newtonian and the nonnewtonian regimes for each phase [8]. The considered Young moduli $E$ for anorthite and diopside were $70 \mathrm{GPa}$ and $170 \mathrm{GPa}$, respectively. The Young $E_{*}$ and shear $\mu_{*}$ moduli of the equivalent medium was calculated by the proposed in [18] approach because of strong contrast of inclusions and matrix properties. 


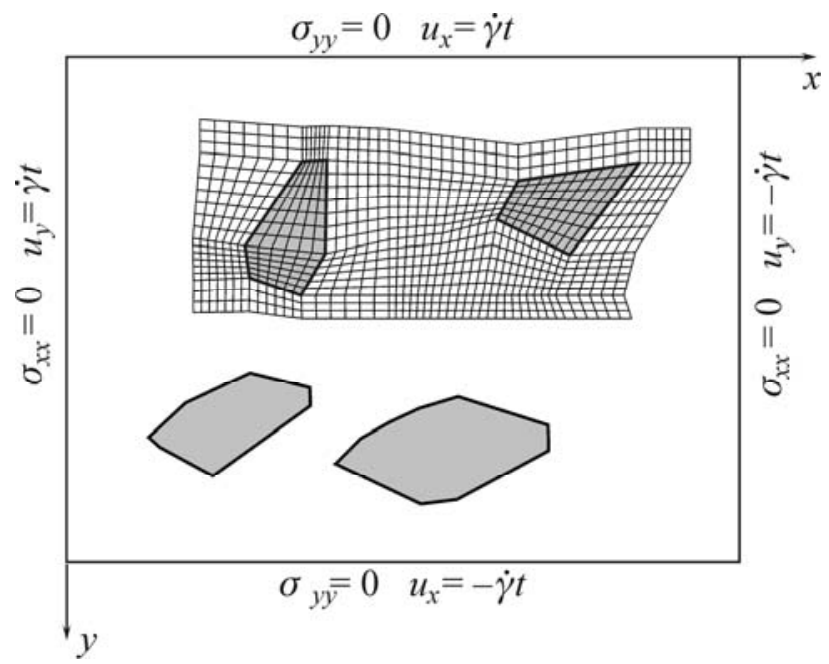

Fig. 3. The calculation domain, boundary conditions and fragment of finite element mesh of the typical representative elementary volume

The REV was incrementally deformed in shear up to $\gamma_{0}=0.02$ at the strain rate $\dot{\gamma}=0.0001 \mathrm{~s}^{-1}$, which is close to our experimental condition and still corresponds macroscopically to the GBS regime (Fig. 1b). In Fig. 4 we show computed mechanical fields reduced to $\tau_{0}=\mu_{*} \gamma_{0}$ at time moment $t=200$ s corresponding to hydrostatic stresses $\sigma$ (Fig. $4 a$ ) and the equivalent shear stresses $\tau$ (Fig. $4 b$ ). The latter figure shows the value $\tau$ affecting the embedding effective material and the embedded actual composite. The stress level appears nearly homogeneous in the embedding material, whilst the most important fluctuations are observed within the embedded inclusions bearing material.

The following simulation has shown that whilst the far-field effective medium is macroscopically dominated by GBS, locally the heterogeneous two-phase microstructure induces strong disparities in terms of stresses and strain rates. The results show a mixed type of behavior, combining areas dominated respectively by GBS or by CSP, which actually corresponds to our experimental observations.
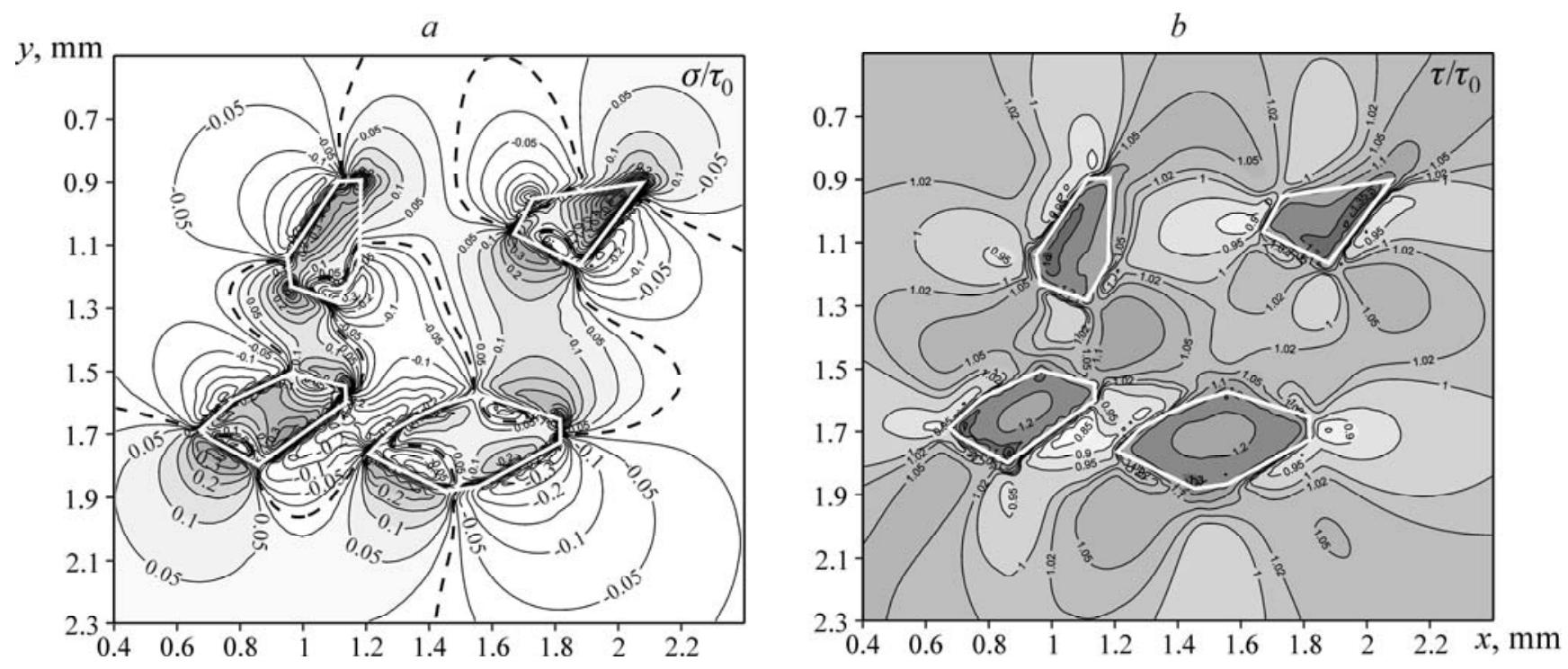

Fig. 4. Hydrostatic stress $\sigma(a)$, shear stress $\tau(b)$ 


\section{CONCLUDING REMARKS}

Gabbroic two-phase rock sample composed of diopside rigid inclusions dispersed in a ductile anorthite matrix were deformed in torsion up at high confining pressure and temperature. The macroscopic flow behavior was linear-viscous, related to diffusion mediated grain sliding mechanisms. However, at the local scale, in the vicinity of and within the diopside inclusions we observed several concomitant deformation/accommodation mechanisms, such as dislocation creep, recrystallization, and micro-fracturing. But, at the experimental conditions these mechanisms necessitate substantially higher stresses (above $150 \mathrm{MPa}$ ) than the overall supported flow stress [8], which in turns evidence local stress enhancement in relation with the heterogeneous nature of the two-phase material.

Numerical simulations of the shear deformation process evidenced that the local stresses may be enhanced by a factor of $2-5$, in relation with the heterogeneous nature of the two-phase material in terms of microstructure, strength contrasts, inclusion shapes and their local interactions. This finding is fully consistent with our observations of the triggering of local dislocation creep processes and the marginal recrystallization of the diopside inclusions.

Our study questions the relevance of paleopiezometry based on dislocation densities, sub-grain and recrystallized grain sizes in such materials. Indeed, the very important local fluctuations of stresses related to the heterogeneous microstructure would probably result in overestimations of the overall flow stress supported by the shear zone. Besides, our findings indicate that the overall flow behavior would be dominated by the fine grained matrix and the related GBS processes, and hence that the mylonitic high temperature shear zones should be modeled on the basis of Newtonian rheology, with correspondingly low flow stresses.

Lastly, our observations indicate that the volumetrically dominant grain sliding mechanisms operating in the ultramylonitic rocks could result in grain scale cavitation, cavitation coalescence and damage, and ultimately in ductile failure, which may also propagate through the stronger wall rock. Such phenomena may therefore trigger deep seismic events within nominally aseismic ductile shear zones.

The study was conducted with partial support from the Russian Foundation for Basic Research, Grant No. 09-05-00975.

\section{REFERENCES}

1. R. Bürgmann and G. Dresen, "Rheology of the lower crust and upper mantle: evidences from rock mechanics, geodesy, and field observations," Annual Rev. Earth Planet. Sci., 36 (2008).

2. Tullis and Yund, "Dynamic recrystallization of feldspar: a mechanism for ductile shear zone formation," Geology, 13, No. 4 (1985).

3. A. M. Boullier and Y. Guéguen, "SP mylonites: origin of some mylonites by superplastic flow," Contr. Mineral. Petrol., 50 (1975).

4. J. H. Behrmann and D. Mainprice, "Deformation mechanisms in a high temperature quartz-feldspar mylonite: evidence for superplastic flow in the lower crust," Tectonophysics, 140 (1987).

5. T. Kenkmann and G. Dresen, "Stress gradients around porphyroclasts: paleopiezometric estimates and numerical modeling," J. Struct. Geol., 20, Nos. 2 and 3 (1998).

6. T. Kenkmann and G. Dresen, "Dislocation microstructure and phase distribution in a lower crustal shear zone - an example from the Ivrea-Zone, Italy," Int. J. Earth Sci., 91, No. 3 (2002).

7. R. Kruse and H. Stunitz, "Deformation mechanisms and phase distribution in mafic high-temperature mylonites from the Jotun Nappe, southern Norway," Tectonophys., 303 (1999).

8. A. Dimanov and G. Dresen, "Rheology of synthetic anorthite-diopside aggregates: implications for ductile shear zones," J. Geophys. Res., 110, B7, B07203 (2005). 
9. R. L. Rudnick and S. R. Taylor, "The composition and petrogenesis of the lower continental crust: a xenolith study," J. Geophys. Res., 92 (1987).

10. M. S. Paterson and D. L. Olgaard, "Deformation tests to large shear strains in torsion," Journal of Structural Geology, 22 (2000).

11. E. Rybacki and G. Dresen, "Dislocation and diffusion creep of synthetic anorthite aggregates," J. Geophys. Res., 105 (2000).

12. A. Dimanov, M. P. Lavie, G. Dresen, et al., "Creep of polycrystalline anorthite and diopside," J. Geophys. Res., 108, B1 (2003).

13. E. Rybacki, M. Gottschalk, and G. Dresen, "Influence of water fugacity and activation volume on the flow properties of fine-grained anorthite aggregates," J. Geophys. Res., 111 (2006).

14. A. Dimanov, E. Rybacki, R. Wirth, and G. Dresen, "Creep and strain-dependent microstructures of synthetic anorthite-diopside aggregates," J. Struct. Geol., 29 (2007).

15. F. Gueydan, Y. M. Leroy, L. Jolivet, and P. Agard, "Analysis of continental midcrustal strain localization induced by reaction-softening and microfracturing," Journal of Geophysical Research, 108, B2 (2003).

16. J. L. Raphanel, G. Ravichandran, and Y. M. Leroy, "Three-dimensional rate-dependent crystal plasticity based on Runge - Kutta algorithms for update and consistent linearization," IJSS, 41 (2004).

17. L. A. Nazarova ad L. A. Nazarov, "Dilatation and the formation and evolution of disintegration zones in the vicinity of heterogeneities in a rock mass," Journal of Mining Science, No. 5 (2009).

18. L. A. Nazarov, L. A. Nazarova, and A. I. Artemova, "Statistic approach to the equivalent modeling of rock masses," Journal of Mining Science, No. 6 (2009). 